\title{
CT-Guided Placement of a Neuromonitoring Suite in Swine for Trauma and Resuscitation Research
}

\author{
Janet Bonin* BS', Hossam Abdou* MD², Joseph Edwards MD², \\ Neerav Patel MD'2, Michael Richmond BS'2, Noha Elansary BS', \\ Kelly Poe BS ${ }^{1}$ and Jonathan J Morrison PhD FRCS ${ }^{2}$ \\ 'University of Maryland School of Medicine, Baltimore, Maryland, USA \\ ${ }^{2} R$ Adams Cowley Shock Trauma Center, University of Maryland Medical System, Baltimore, Maryland, USA
}

\begin{abstract}
Background: In this manuscript, we aim to describe a standardized method for placement of a neuromonitoring suite into the brain of a porcine model using computed tomography (CT) guidance for use in trauma and resuscitation research.

Methods: A baseline CT allowed for precise planning of the placement of the neuromonitoring suite including measurement of skull thickness at the location of the intended burr hole. After the burr hole was drilled, three neuromonitoring probes (pressure catheter, temperature probe, and laser doppler flow probe) were advanced into the brain parenchyma of the swine. A subsequent CT confirmed appropriate placement of the neuromonitoring suite. Results: Effective placement of the neuromonitoring suite was accomplished successfully and without complication in six Yorkshire swine. Mean duration of the procedure was $49.6 \pm 6.3 \mathrm{~min}$. Representative data from one animal include the following presented as mean \pm standard deviation: intracranial pressure of $10 \pm 0 \mathrm{mmHg}$, cerebral perfusion pressure of $61 \pm 1 \mathrm{mmHg}$, intracranial temperature of $34.8 \pm 0^{\circ} \mathrm{C}$, and brain perfusion of $704 \pm 13$ relative perfusion units.

Conclusions: This CT-guided method facilitates placement of a neuromonitoring suite in a safe and reliable manner. The use of a neuromonitoring suite using CT may offer valuable insight into cerebral perfusion in the context of endovascular resuscitation.
\end{abstract}

Keywords: Neuromonitoring; Computed Tomography; Swine Research; Resuscitation Research

\section{INTRODUCTION}

After major hemorrhage from trauma, approximately $40 \%$ of patients die before bleeding can be controlled [1]. Endovascular techniques such as Resuscitative Endovascular Balloon Occlusion of the Aorta (REBOA) and Selective Aortic Arch Perfusion (SAAP) utilize a balloon catheter to obtain hemorrhage control and manipulate central blood pressure and perfusion in an effort

*Co-first authors who contributed equally to the development of this manuscript.

\section{Corresponding author:}

Jonathan J Morrison, PhD, FRCS, R Adams Cowley Shock Trauma Center, 22 S. Greene Street, Baltimore, Maryland, USA 21201.

Email: jonathan.morrison@som.umaryland.edu

(C) 2021 CC BY 4.0 - in cooperation with Depts. of Cardiothoracic/ Vascular Surgery, General Surgery and Anesthesia, Örebro University Hospital and Örebro University, Sweden to resuscitate patients [2]. Although these methods can be effective in preventing further hemorrhage, it remains unclear how these interventions affect brain perfusion. It is understood from cardiac arrest patients that both hypoperfusion and reperfusion of brain tissue can cause irreversible neuronal damage [3]. It is critical to develop reliable methods of neuromonitoring in order to assess neurological indices in the setting of novel endovascular resuscitation techniques.

Placement of neuromonitoring probes into the porcine model has traditionally been performed using anatomical landmarks such as the bregma [4]. Measuring the distance from certain bony landmarks, such as the distance from the lateral to frontal suture and anterior to the coronal suture, allow approximate triangulation for selection of probe location [5]. However, these measurements only offer an estimate of intraparenchymal probe location. Precise and consistent placement of the 
probe into the intended brain region is critical due to the interlobar and even intralobar variation in porcine cerebral blood flow [6]. Consistent probe placement across subjects would assure true comparison between the same brain regions.

Neuromonitoring probe placement can now be directed using imaging techniques such as computed tomography (CT) [7]. Imaging allows for improved precision and confirmation of appropriate probe placement. This method is becoming increasingly practical with improving technology and the availability of portable CT scanners. The aim of this manuscript is to describe a standardized method of CT-guided placement of a neuromonitoring suite in a porcine model.

\section{METHODS}

\section{Overview}

Procedures were approved by the University of Maryland, Baltimore Institutional Animal Care and Use Committee (Approval \#0320017) and conformed to National Institutes of Health guidelines for ethical animal research. Six adult male Yorkshire swine weighing between $60 \mathrm{~kg}$ and $80 \mathrm{~kg}$ were enrolled in the study, which utilized ADInstruments devices, including pressure catheters, temperature probes, and laser doppler flow (LDF) probes (ADInstruments, Sydney, Australia). Insertion consisted of the following steps: preparation, burr hole creation, and insertion of neuromonitoring probes.

\section{Preparation}

The evening before the procedure, animals had free access to water but were fasted from food. Animals were sedated using intramuscular injection of Telazol $(5$ $\mathrm{mg} / \mathrm{kg}$ ) and Xylazine $(2 \mathrm{mg} / \mathrm{kg})$, then placed in ventral recumbency on the operating table. Endotracheal intubation was attained, and anesthesia was maintained with isoflurane $(1-3 \%)$. Hair was removed from the scalp, and the site was scrubbed with povidone-iodine; after drying, the operative site was draped in a sterile fashion.

\section{Burr Hole Creation}

Creation of a burr hole was performed under CT guidance using an OmniTom portable CT scanner (Neurologica, Danvers, USA) and a drill (Ryobi, Hiroshima, Japan). CT images were acquired with 1.25-mm axial slices, $120 \mathrm{kV}$, and $40 \mathrm{~mA}$. The protocol was designed to maximize the power of the machine to optimize images in the setting of the thick skull of swine. Images were transferred to a picture archiving and communication system (PACS) (Purview, Annapolis, USA) for burr hole planning.
A baseline non-contrasted CT was obtained, and images transferred to PACS. Skull thickness was measured $1 \mathrm{~cm}$ posterior and lateral to the bregma, where the creation of a burr hole was intended. A drill bit depth stopper was placed onto the drill bit (9/32 in) at this level to prevent drilling beyond the skull. A scalpel was used to make a cruciate incision at the site of intended burr hole creation. The drill, with depth-limiting stopper in place, was used to create a burr hole.

\section{Insertion of Neuromonitoring Probes}

An 18-gauge needle was inserted through the burr hole into the brain parenchyma, and a $1.8-\mathrm{Fr}$ pressure catheter was advanced through the needle into the brain. The needle was slowly retracted to expose the catheter's pressure sensor. A 20-gauge needle was placed through the burr hole for placement of the temperature probe following the same procedure as for the pressure catheter. The LDF probe was directly advanced into the brain parenchyma. Figure $1 a$ exhibits all neuromonitoring probes in place. A CT scan was performed to confirm the location of the probes in the brain parenchyma (Figure $1 b$ ).

Vitals were monitored throughout the experiment. At the conclusion, animals were euthanized using potassium chloride while under inhalant anesthesia. All data were captured continuously in LabChart (ADInstruments, Sydney, Australia). Data were averaged over a period of $7 \mathrm{~min}$ and is presented as mean \pm standard deviation.

\section{RESULTS}

The neuromonitoring suite was placed successfully and without complication in all six animals. The mean weight was $67.1 \pm 4.9 \mathrm{~kg}$. The mean duration from procedure start to start of data collection was $49.6 \pm 6.3$ min. Intracranial and relevant hemodynamic indices for a representative animal are presented in Figure $1 c$ and Table 1.

\section{DISCUSSION}

This study outlines a standardized method for CT-guided placement of a neuromonitoring suite in the porcine model. This method was demonstrated to be a reliable and safe procedure in all animals and enables laboratories to collect several intracranial indices in a dependable manner. CT can be used to confirm that intracranial probes are situated appropriately and allows for adjustment as needed for precise placement of probes as desired. No complications were encountered using the described method.

Neuromonitoring probe placement has typically been performed using anatomical landmarks, but the natural variance among individuals presents a potential 


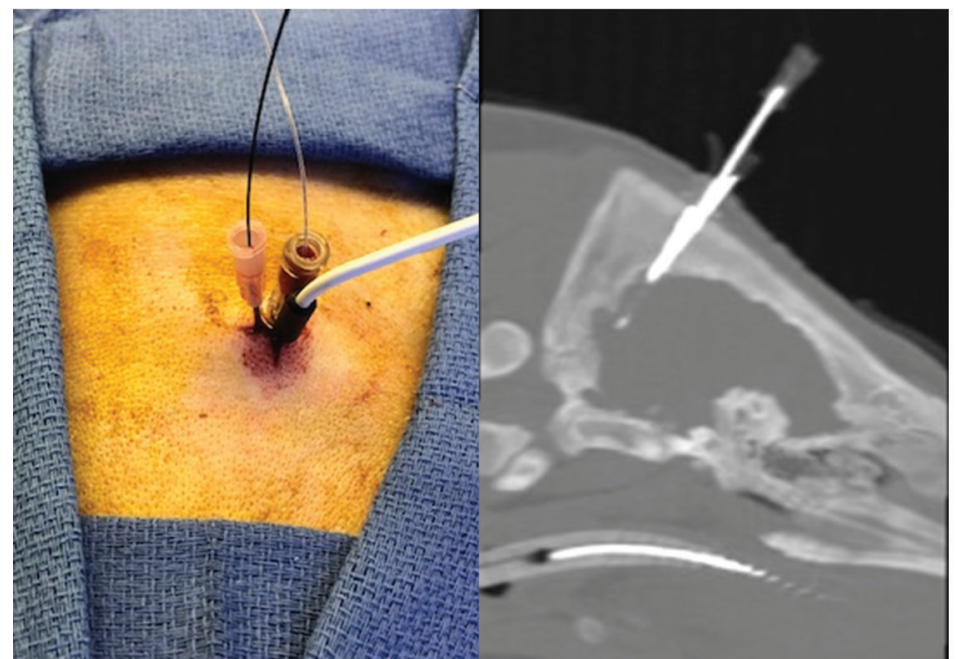

(a)

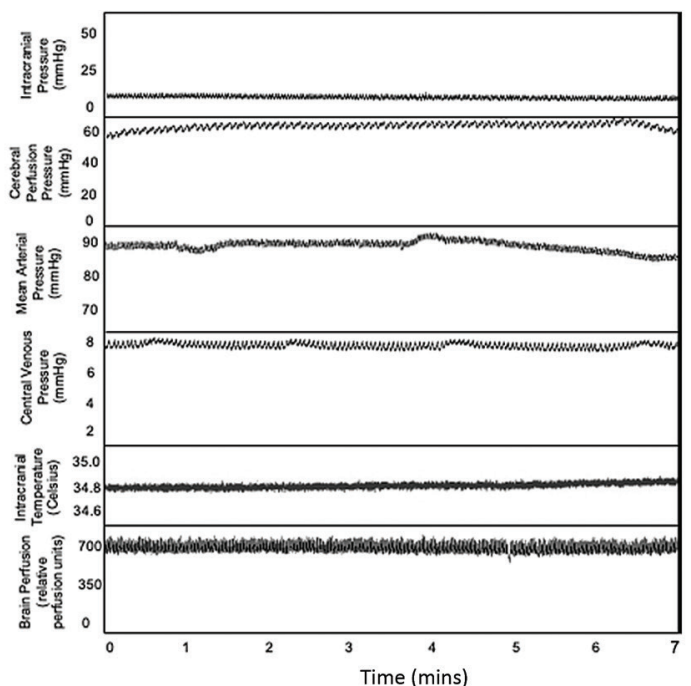

(c)

Figure 1 Neuromonitoring suite in place and representative data captured. (a) Image of burr hole with neuromonitoring probes in place. (b) Example of CT confirming placement of neuromonitoring suite. (c) Graph of representative neuromonitoring data.

Table 1 Representative data collected using this neuromonitoring suite.

\begin{tabular}{lc}
\hline & Mean $\pm S D$ \\
\hline Intracranial pressure $(\mathrm{mmHg})$ & $9.93 \pm 0.13$ \\
Cerebral perfusion pressure $(\mathrm{mmHg})$ & $61.02 \pm 1.11$ \\
Mean arterial pressure $(\mathrm{mmHg})$ & $90.7 \pm 0.10$ \\
Central venous pressure $(\mathrm{mmHg})$ & $7.79 \pm 0.13$ \\
Intracranial temperature $\left({ }^{\circ} \mathrm{C}\right)$ & $34.80 \pm 0.01$ \\
Brain perfusion (relative perfusion units) & $703.50 \pm 13.20$ \\
\hline
\end{tabular}

SD: standard deviation.

confounder. The increased availability of advanced imaging technology offers an opportunity to improve precision of instrumentation in order to gather more consistent data.

This method of placing a neuromonitoring suite can be a valuable adjunct in trauma and resuscitation research to evaluate neurological parameters such as brain perfusion during and following resuscitative maneuvers. The concept of endovascular resuscitation has grown over the last several years with the emergence of multiple novel techniques, some of which have demonstrated promising results in achieving return of spontaneous circulation. However, data are lacking with regard to brain perfusion and neurological recovery in the setting of these techniques. The method outlined in this manuscript offers a reliable procedure for instrumenting the porcine brain that allows future researchers to effectively evaluate brain perfusion in the setting of resuscitation. This information will prove critical as we continue to assess novel techniques and how to best utilize them to serve our patients in order to attain not only hemodynamic recovery but also meaningful neurological recovery.

\section{CONCLUSIONS}

CT-guided placement of a neuromonitoring suite is a safe and reliable procedure in the swine model. This technique enables researchers to further explore crucial neurological parameters, which will be especially useful in trauma and resuscitation research, particularly in evaluating novel endovascular resuscitation techniques in their ability to provide adequate brain perfusion to allow for meaningful neurological recovery.

\section{Ethics Statement}

(1) All the authors mentioned in the manuscript have agreed to authorship, read and approved the manuscript, and given consent for submission and subsequent publication of the manuscript.

(2) The authors declare that they have read and abided by the JEVTM statement of ethical standards including rules of informed consent and ethical committee approval as stated in the article.

\section{Conflicts of Interest}

The authors declare that they have no conflicts of interest.

\section{Funding}

The authors disclosed receipt of the following financial support for the research, authorship, and/or publication of 
this article: This work was supported by the Department of Defense [grant \#HU0001-19-2-0072, sub-award \#4798].

\section{Author Contributions}

All authors have substantially contributed to the study and manuscript writing.

\section{REFERENCES}

[1] Borger van der Burg BLS, van Dongen TTCF, Morrison JJ, et al. A systematic review and meta-analysis of the use of resuscitative endovascular balloon occlusion of the aorta in the management of major exsanguination. Eur J Trauma Emerg Surg. 2018;44:535-50.

[2] Madurska MJ, Ross JD, Scalea TM, et al. State of the Art Review - Endovascular Resuscitation. Shock; 2021; In press.
[3] van den Brule JMD, van der Hoeven JG, Hoedemaekers CWE. Cerebral perfusion and cerebral autoregulation after cardiac arrest. BioMed Res Int.2018;2018:4143636.

[4] Mangla S, Choi JH, Barone FC, et al. Endovascular external carotid artery occlusion for brain selective targeting: a cerebrovascular swine model. BMC Res Notes. 2015;8:1-6.

[5] Andrade AF de, Soares MS, Patriota GC, et al. Experimental model of intracranial hypertension with continuous multiparametric monitoring in swine. Arq Neuropsiquiatr. 2013;71:802-6.

[6] Delp MD, Armstrong RB, Godfrey DA, et al. Exercise increases blood flow to locomotor, vestibular, cardiorespiratory and visual regions of the brain in miniature swine. J Physiol. 2001;533:849-59.

[7] Platt SR, Holmes SP, Howerth EW, et al. Development and characterization of a Yucatan miniature biomedical pig permanent middle cerebral artery occlusion stroke model. Exp Transl Stroke Med. 2014;6:5. 